\title{
Biocompatibility of intracortical microelectrodes: current status and future prospects
}

\author{
Cristina Marin ${ }^{1}$ and Eduardo Fernández ${ }^{1,2 *}$ \\ Institute of Bioengineering, University Miguel Hernández, Elche, Spain \\ 2 Center for Biomedical Research Network in Bioengineering, Biomaterials and Nanomedicine, Elche, Spain
}

Edited by:

Martin Stelzle, University of Tübingen, Germany

\section{Reviewed by:}

David Martin, University of Michigan, USA

Martin Stelzle, University of Tübingen, Germany

\section{${ }^{*}$ Correspondence:}

Eduardo Fernández, Institute of

Bioengineering, Universidad Miguel Hernández, Avda. Universidad s/n,

Elche 03202, Alicante, Spain.

e-mail:e.fernandez@umh.es

\begin{abstract}
Rehabilitation of sensory and/or motor functions in patients with neurological diseases is more and more dealing with artificial electrical stimulation and recording from populations of neurons using biocompatible chronic implants. As more and more patients have benefited from these approaches, the interest in neural interfaces has grown significantly. However an important problem reported with all available microelectrodes to date is long-term viability and biocompatibility. Therefore it is essential to understand the signals that lead to neuroglial activation and create a targeted intervention to control the response, reduce the adverse nature of the reactions and maintain an ideal environment for the brain-electrode interface. We discuss some of the exciting opportunities and challenges that lie in this intersection of neuroscience research, bioengineering, neurology and biomaterials.
\end{abstract}

Keywords: neural prosthesis, neural interface, brain-machine interface, coating, glial scar

\section{INTRODUCTION}

The treatment of neurologic disorders and the restoration of lost function by neuroprosthetic devices has grown rapidly in the latest years. For example, deep brain stimulators have been implanted successfully in patients for pain management and for control of motor disorders such as Parkinson's disease (Stieglitz et al., 2004; Canavero and Bonicalzi, 2007); cochlear implants are being used for restoring auditory function and micro-array type devices have been implanted in rudimentary artificial vision systems (Fernandez et al., 2005; Merabet et al., 2007; Normann et al., 2009). Moreover advances in artificial limbs and brain-machine interfaces are now providing hope of increased mobility and independence for amputees and paralyzed patients and there is preliminary data showing that by using electrophysiological methods it is possible to extract information about intentional brain processes and then translate these signals into models that are able to control external devices (Hochberg et al., 2006; Donoghue et al., 2007; Truccolo et al., 2008). As more and more patients have benefited from this approach, the interest in neural interfaces has grown significantly. However an important problem reported with all available neural electrodes to date, is long-term viability and stability.

All neural probes need a stable electronic/neural interface that enables selective recording and/or activation of specific groups of neurons without deterioration of the electrodes or surrounding neural tissue. To optimally record from or excite neurons, it is important to position the microelectrodes very close to the neurons we are trying to interact with (within a few microns). This means that the electrodes must be located deep within the nervous system and have dimensions that are similar to the size of the neurons. Other important attributes are:

- Special design for insertion in any part of cerebral cortex, including sulci of highly folded cortices such as those of humans.

- Reaching neurons at the desired three-dimensional location.
- Bi-directional communication with neurons and ensembles of neurons.

- Providing appropriate and stable electrical interfacing.

- Minimizing tissue damage and scarring.

- Rendering the devices as inert as possible from the biocompatibility, biostability and biofouling standpoints.

Following these basic requirements great efforts have been made to develop penetrating multielectrode arrays, with dimensions of the same order of magnitude as the cortical cells that can be used to excite neurons more selectively and with electrical currents much smaller than those used by surface electrodes (Normann et al., 1999). The two main dominated approaches are multiple insulated metal microwires (Kralik et al., 2001; Carmena et al., 2005; Nicolelis, 2005; Musallam et al., 2007; Ganguly et al., 2009; Nicolelis and Lebedev, 2009) and micromachined penetrating microelectrode arrays with various substrate materials, insulating dielectrics, and substrate shaping technologies than can be flexible and based on polymers (Stieglitz and Meyer, 1999; Rousche et al., 2001; Wise, 2005; Chen et al., 2009; Kozai and Kipke, 2009) or more rigid such as the Utah's array (Normann, 2007), the Michigan array (Seymour and Kipke, 2007) or the new NeuroProbes arrays (Neves, 2007).

Factors affecting brain tissue reactions to the implanted neural probes include the mechanical trauma during insertion, foreign body reaction, implantation method and physical properties of the electrodes. Thus there are acute and chronic inflammatory reactions which affect both the neural tissue and the surface of the microelectrodes (Hoogerwerf and Wise, 1994; Woodford et al., 1996; Heiduschka and Thanos, 1998; Agnew et al., 1999; Liu et al., 1999; Majji et al., 1999; Turner et al., 1999; McCreery et al., 2002). These reactions often result in damage of neural elements and microelectrodes and lead to the proliferation of a glial scar around the implanted spikes which prevents neuronal fibers to be recorded or stimulated (Fawcet and Asher, 1999; Turner et al., 1999). As a 
consequence one of the major prerequisites for the application of neural probes is that the organism accepts the implant with minimal tissue response (see next section).

\section{CURRENT CONCEPTS OF BIOCOMPATIBILITY OF NEURAL PROBES}

Biocompatibility is used extensively within biomaterial science, but there still exists a great deal of uncertainty about what it actually means and about the mechanisms that are subsumed within the phenomena that collectively constitute the biocompatibility (Williams, 2008). Biocompatibility has traditionally been concerned with aspects of biological, chemical and physical properties of the implant. This implies that the probes do not evoke a toxic or immunologic reaction, do not harm or destroy enzymes, cells or tissues, do not compress adjacent tissues inducing vascular problems and be able to remain for a long term within the organism without encapsulation or rejection (Heiduschka and Thanos, 1998).

To those who were developing and using the first generation of implantable devices (1940-1980) it was becoming increasingly obvious that the best performance was achieved with materials that were the least reactive chemically (Williams, 2008). However, while the surface composition of the implant is an important parameter, in some cases physical properties (size, shape, stiffness) are the major determinants of biocompatibility (Edell et al., 1992; Heiduschka and Thanos, 1998; Ward et al., 2009). Furthermore although it is widely accepted to define biocompatibility as "the ability of a material to perform with an appropriate host response in a specific application", nowadays any neural probe is comprised of more than one material, therefore we have to move from a material base to an specific application base definition. Consequently a neural implant can be considered to be biocompatible if,
- It performs its desired function without eliciting any undesirable local or systemic effect in the recipient of the implant or in the own implant materials.

- It remains for a long term within the organism, entirely functional and with the desired degree of incorporation in the host.

This concept is not limited to minimize local lesions, but also encloses the whole behavior of the implant in its biological environment (Walter et al., 1999; Kohler et al., 2001). Therefore three areas have to be considered, the "biosafety", the "biofunctionality" and the "biostability". Biosafety means that the implant does not harm its host in any way, biofunctionality is related with the ability of the device to perform its intended function, and biostability means that the implant must not be susceptible to attack of biological fluids, proteases, macrophages or any substances of the metabolism (Heiduschka and Thanos, 1998). In addition it should also be taken into account the "biotolerability" or the ability of the implant to reside in the body for long periods of time with only low degrees of inflammatory reaction. All these considerations imply extreme demands on stability and function of neural implants and place unique constraints on the architecture, materials, and surgical techniques used in the implementation of intracortical microelectrodes.

\section{REACTIVE RESPONSES AROUND NEURAL PROBES}

Implantation of any neural probe is always a traumatic procedure. Thus, when a neural probe is inserted into the brain, some neurons and glial cells are killed or injured during insertion, blood vessels are disrupted, and the blood-brain barrier is damaged (Figure 1A). This acute injury triggers a large network of morphological and metabolic changes such as the release of cytokines and neurotoxic
A
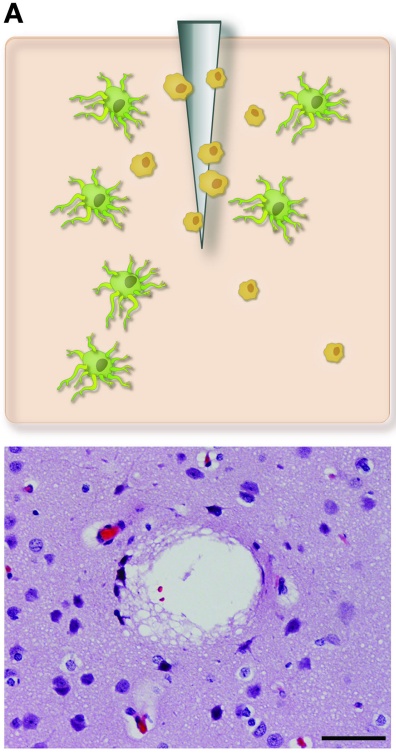

B
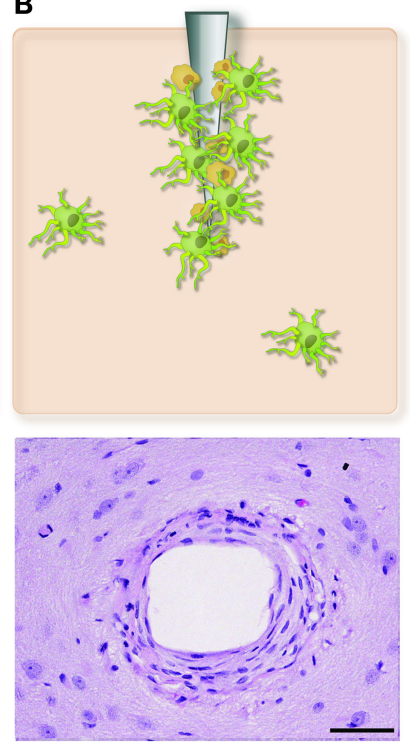

C

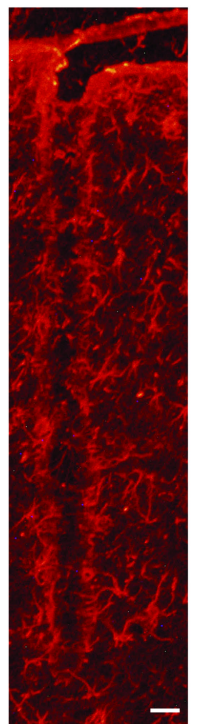

D

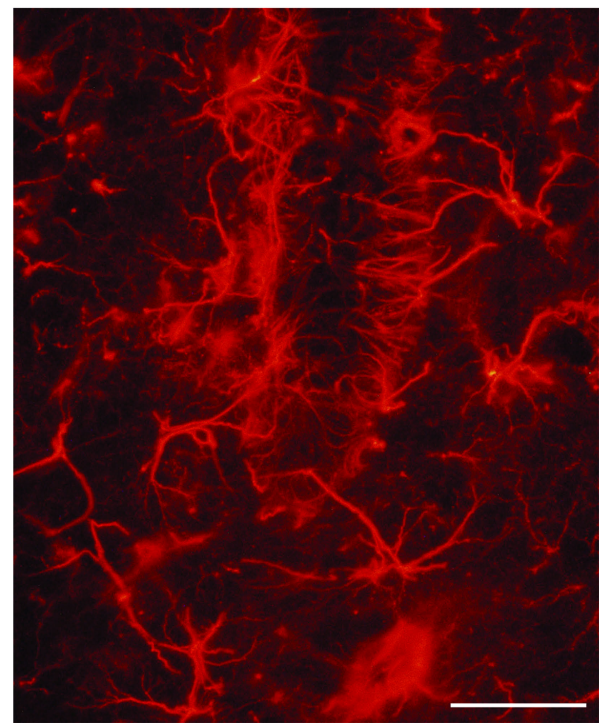

FIGURE 1 | Development of glial encapsulation on an intracortical microelectrode. (A) Acute neural injury caused by inserting a microelectrode into the brain cortex. Astrocytes and microglial cells become activated and migrate to the site of injury. (B) Chronic response showing a dense sheath around implanted probes, which contains fibroblasts, macrophages and astrocytes. (C,D) The reactive astrocytes, immunohistochemically labeled here for GFAP, encapsulate the neural probes forming a dense cellular sheath. Calibration bar $=50 \mu \mathrm{m}$. 
free radicals, invasion of blood-borne macrophages and edema. The damaged tissue assumes a state of emergency, rapidly changing its gene expression and stimulating nearby microglia and astrocytes (Lee et al., 2005; Polikov et al., 2005; Biran et al., 2007; Grill et al., 2009; McConnell et al., 2009). This mechanically induced wound healing response appears to be of transitory nature since electrode tracks could not be found in animals after several months when the electrodes are inserted and quickly removed (Polikov et al., 2005).

We should be aware that this acute response plays an important role in two crucial physiological processes: protection against infectious agents and repair of the damaged tissue. Thus the initial activation and migration of microglial cells are likely beneficial and include production of neurotrophic substances and cell adhesion molecules, which support injured neurons and appear necessary for restorative events to take place (Eddleston and Mucke, 1993). However, this largely beneficial initial phase usually results in a more adverse long-term response that is dependent of the extension of the injury. Therefore these reactions often result in damage of neurons and microelectrodes and lead to the proliferation of a glial scar around the implanted probes (Figure 1) which prevents neurons to be recorded or stimulated (Polikov et al., 2005; Seymour and Kipke, 2007; McConnell et al., 2009). In this way the outer surface of the neural probes is frequently surrounded by a dense sheath, which contains markers of macrophages, astrocytes and fibroblasts (Kim et al., 2004). Furthermore in addition to the astroglial encapsulation, there is a persistent inflammatory response at the microelectrode-brain tissue interface, with abundant activated macrophages and a loss of neurons and their projections in the brain tissue immediately surrounding the microelectrodes (Figure 2), that either die or migrate away from the interface (Biran et al., 2005). This long-term response is of concern because increases tissue impedance, difficult the regrowth of neuronal processes and diminishes the ability to record and stimulate in long-term applications. In this context it is important to emphasize that not only

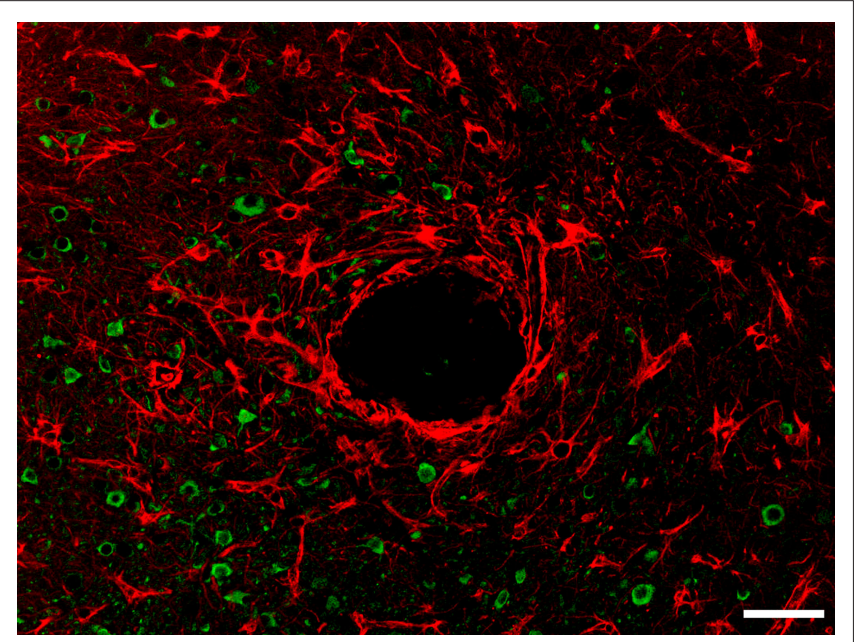

FIGURE 2 | Photomicrograph showing astrocytes (GFAP staining, red) and neurons (NeuN staining, green) around one microelectrode track (asterisk) following chronic implantation of a NeuroProbes multielectrode array in rabbit occipital cortex. Calibration bar $=50 \mu \mathrm{m}$. must neurons be alive, but they must also have normal dendritic and axonal trees, normal network connectivity and functional cell bodies (McConnell et al., 2009).

\section{RE-ENGINEERING THE NEURAL INTERFACE}

Recent studies on the effects of neural injury following the insertion of microelectrodes suggest that the neuroglial reaction is a graded, stereotypic response that can be controlled (Polikov et al., 2005; Moxon et al., 2007; Zhong and Bellamkonda, 2007; Grill et al., 2009). In general, the neural response has two parts, a short- and a long-term response. If the short-term response can be controlled, it appears likely that the reactive gliosis and other long-term inflammatory processes around neural probes can be prevented.

Multiple strategies have been devised to regulate glial scar formation. For example by using controlled molecular engineering it may be possible to reduce the tissue reaction around neural probes as well as to maximize the beneficial aspects of the response and to minimize adverse responses. One approach to modulate the inflammatory response and achieve better integration of the neural probes with brain tissue is to develop new coatings that modify the neural probe surfaces. A first kind of surface modifications that can be done is the preparation of smooth or rough surfaces, but the most widely used method to enhance biocompatibility is the chemical modification of neural probe surfaces with anti-inflammatory compounds, adhesion proteins, or bioactive molecules (Heiduschka and Thanos, 1998; He et al., 2006; Ludwig et al., 2006; Moxon et al., 2007; Rennaker et al., 2007; Seymour and Kipke, 2007; Zhong and Bellamkonda, 2007; Leung et al., 2008; Williams, 2008; Grill et al., 2009). The portfolio of possible chemical modifications also include, depending of the kind of molecules bound to the surface, biological modifications such as coating with hyaluronic acid, peptides, sugars or growth factors (Heiduschka and Thanos, 1998; Dodla and Bellamkonda, 2006; Azemi et al., 2008; Dodla and Bellamkonda, 2008) and non-biological modifications as hydrogels (Crompton et al., 2007; Frampton et al., 2007; Kim et al., 2010), polymers (Ludwig et al., 2006; Stice et al., 2007; Asplund et al., 2009; Richardson et al., 2009) and carbon nanotubes (Lovat et al., 2005; Mazzatenta et al., 2007; Pancrazio, 2008; Gabriel et al., 2009).

Aside from coating the electrodes with different biomolecules, another method to control the glial response is to fabricate neural probes with different physical dimensions and geometries (Stice et al., 2007; Ward et al., 2009). However, although some studies suggest that neural probe geometry is an important parameter for reducing chronic tissue encapsulation (Seymour and Kipke, 2007) others found that device insertion promotes two responses: an early response that is proportional to device size and a sustained response that is independent of device size, geometry, and surface roughness and is more likely due to tissue-device interactions (Szarowski et al., 2003).

The quality of the surgical procedure also plays a decisive role for successfully long-term applications and deficiencies in surgical procedures may likely contribute to most of the difficulties in chronic experiments. Thus careful implantation prevents cortical compression and also seems to increase the longevity and stability of intracortical microelectrodes (Nicolelis et al., 1999; Rennaker et al., 2005). 


\section{CHALLENGES AND FUTURE PERSPECTIVES}

Understanding the interaction between the implanted device and the local cellular environment is critical for improving the integration and function of any neural interface. This response needs to be controlled to ensure the function of the implant and the desired degree of incorporation in the host, without eliciting any undesirable local or systemic effect. Consequently some of the main scientific challenges are:

(1) Characterization of new coating technologies and biocompatible coating materials (e.g. peptides similar to extracellular matrix and hybrid polymers with adjustable electrical properties) for improving the biocompatibility and electrochemical properties of neural interfaces.

(2) Improve long-term biocompatibility and re-engineer the brain's response to the insertion of microelectrodes through controlled molecular engineering to find a balance that minimizes any possible adverse response and enhances the trophic responses.

For future developments, it would be desirable to promote neuronal growth toward electrodes and find special coverings for the different parts of the neuroprosthetic devices (recording electrodes, stimulating electrodes, insulation material). These coatings (Figure 3) will be able to induce adhesion, repulsion, activation or deactivation of specific cell types upon contact with the modified surface promoting a good incorporation into the tissue and helping to avoid undesirable responses of the immune system and the damage of the microelectrodes.

In the longer term, several neurological pathologies will be alleviated by local microelectrodes, but keeping these devices biologically and electrically viable for many years still remains a difficult problem. In this context an investigation of the submicron interface between devices and nervous system cells is essential for a better understanding of the continued reaction of the human body to any artificial implant, no matter how apparently inert the material is. We expect that the intersection of neuroscience research, bioengineering, and biomaterials together with intimate collaborations among basic scientists, engineers and clinicians will help to control the neural

\section{REFERENCES}

Agnew, B. J., Duman, J. G., Watson, C. L., Coling, D. E., and Forte, J. G. (1999). Cytological transformations associated with parietal cell stimulation: critical steps in the activation cascade. J. Cell. Sci. 112, 2639-2646.

Asplund, M., Thaning, E., Lundberg, J., Sandberg-Nordqvist, A. C., Kostyszyn, B., Inganas, O., and von Holst, $\mathrm{H}$. (2009). Toxicity evaluation of PEDOT/ biomolecular composites intended for neural communication electrodes. Biomed. Mater. 4, 45009.

Azemi, E., Stauffer, W. R., Gostock, M. S., Lagenaur, C. F., and Cui, X. T. (2008). Surface immobilization of neural adhesion molecule L1 for improving the biocompatibility of chronic neural probes: in vitro characterization. Acta Biomater. 4, 1208-1217.
Biran, R., Martin, D. C., and Tresco, P. A. (2005). Neuronal cell loss accompanies the brain tissue response to chronically implanted silicon microelectrode arrays. Exp. Neurol. 195, 115-126.

Biran, R., Martin, D. C., and Tresco, P. A. (2007). The brain tissue response to implanted silicon microelectrode arrays is increased when the device is tethered to the skull. J. Biomed. Mater. Res. A. 82, 169-178.

Canavero, S., and Bonicalzi, V. (2007). Central pain syndrome: elucidation of genesis and treatment. Expert Rev. Neurother. 7, 1485-1497.

Carmena, J. M., Lebedev, M. A., Henriquez, C. S., and Nicolelis, M. A. (2005). Stable ensemble performance with single-neuron variability during reaching movements in primates. $J$. Neurosci. 25, 10712-10716.

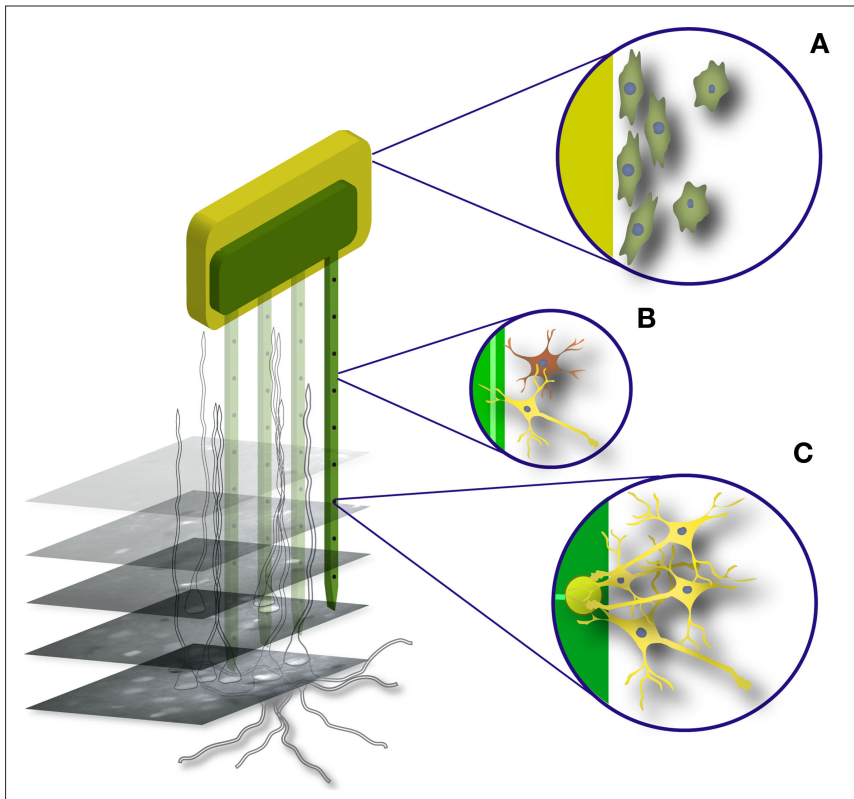

FIGURE 3 | Summary of optimum surface behavior for an implanted neural probe. (A) Surface of encapsulation and insulation material: adsorption of proteins and adhesion of fibroblast for good incorporation into the tissue, no reaction of macrophages. (B) Surface without electrodes: good contact to neurons and glial cells, no reaction of macrophages. (C) Surface of electrodes: attraction and good contact to neurons, no adhesion of fibroblasts, no macrophage reaction.

tissue damage induced by these intracortical microelectrodes and allow us to achieve the therapeutic benefits envisioned by these neural interfaces.

\section{ACKNOWLEDGMENT}

We would like to thank Markus Bongard for all his help with the figures. This work has been supported in part by grants SAF200803694 and CIBER-BBN from the Spanish Government, by the ONCE (National Organization of the Spanish Blind) and by the European Commission through the project "NEUROPROBES IST-027017".
Chen, Y. Y., Lai, H. Y., Lin, S. H., Cho, C. W., Chao, W. H., Liao, C. H., Tsang, S. Chen, Y. F., and Lin, S. Y. (2009). Design and fabrication of a polyimide-based microelectrode array: application in neural recording and repeatable electrolytic lesion in rat brain. J. Neurosci. Methods 182, 6-16.

Crompton, K.E., Goud, J.D., Bellamkonda, R. V., Gengenbach, T. R., Finkelstein, D. I., Horne, M. K., and Forsythe, J. S (2007). Polylysine-functionalised thermoresponsive chitosan hydrogel for neural tissue engineering. Biomaterials 28, 441-449.

Dodla, M. C., and Bellamkonda, R. V (2006). Anisotropic scaffolds facilitate enhanced neurite extension in vitro. J. Biomed. Mater. Res. A. 78, 213-221.

Dodla, M. C., and Bellamkonda, R. V. (2008). Differences between the effect of anisotropic and isotropic laminin and nerve growth factor presenting scaffolds on nerve regeneration across long peripheral nerve gaps. Biomaterials 29, 33-46.

Donoghue, J. P., Nurmikko, A., Black, M., and Hochberg, L. R. (2007). Assistive technology and robotic control using motor cortex ensemble-based neural interface systems in humans with tetraplegia. J. Physiol. (Lond.) 579, 603-611.

Eddleston, M., and Mucke, L. (1993). Molecular profile of reactive astrocytes-implications for their role in neurologic disease. Neuroscience 54, 15-36.

Edell, D. J., Van Toi, V., McNeil, V. M., and Clark, L. D. (1992). Factors influencing the biocompatibility of insertable silicon microshafts in cerebral 
cortex. IEEE Trans. Biomed. Eng. 39, 635-643.

Fawcet, J. W., and Asher, R. A. (1999). The glial scar and central nervous system repair. Brain Res. Bull. 49, 377-391.

Fernandez, E., Pelayo, F., Romero, S., Bongard, M., Marin, C., Alfaro, A., and Merabet, L. (2005). Development of a cortical visual neuroprosthesis for the blind: the relevance of neuroplasticity. J. Neural Eng. 2, R1-R12.

Frampton, J. P., Hynd, M. R., Williams, J. C., Shuler, M. L., and Shain, W. (2007). Three-dimensional hydrogel cultures for modeling changes in tissue impedance around microfabricated neural probes. J. Neural Eng. 4, 399-409.

Gabriel, G., Gomez, R., Bongard, M., Benito, N., Fernandez, E., and Villa, R. (2009). Easily made single-walled carbon nanotube surface microelectrodes for neuronal applications. Biosens. Bioelectron. 24, 1942-1948.

Ganguly, K., Secundo, L., Ranade, G., Orsborn, A., Chang, E. F., Dimitrov, D. F., Wallis, J. D., Barbaro, N. M., Knight, R. T., and Carmena, J. M. (2009). Cortical representation of ipsilateral arm movements in monkey and man. J. Neurosci. 29, 12948-12956.

Grill, W. M., Norman, S. E., and Bellamkonda, R. V. (2009). Implanted neural interfaces: biochallenges and engineered solutions. Annu. Rev. Biomed. Eng. 11, 1-24.

He, W., McConnell, G. C., and Bellamkonda, R. V. (2006). Nanoscale laminin coating modulates cortical scarring response around implanted silicon microelectrode arrays. J. Neural Eng. 3, 316-326.

Heiduschka, P., and Thanos, S. (1998). Implantable bioelectronic interfaces for lost nerve functions. Prog. Neurobiol. 55, 433-461.

Hochberg, L. R., Serruya, M. D., Friehs, G. M., Mukand, J.A., Saleh, M., Caplan, A. H., Branner, A., Chen, D., Penn, R. D., and Donoghue, J. P. (2006). Neuronal ensemble control of prosthetic devices by a human with tetraplegia. Nature 442, 164-171.

Hoogerwerf, A. C., and Wise, K. D. (1994). A 3D micro-electrode array for chronic neural recording. IEEE Trans. Biomed. Eng. 41, 1136-1146.

Kim, D. H., Wiler, J. A., Anderson, D. J., Kipke, D. R., and Martin, D. C. (2010). Conducting polymers on hydrogelcoated neural electrode provide sensitive neural recordings in auditory cortex. Acta Biomater. 6, 57-62.

Kim, Y. T., Hitchcock, R. W., Bridge, M. J., and Tresco, P. A. (2004). Chronic response of adult rat brain tissue to implants anchored to the skull. Biomaterials 25, 2229-2237.

Kohler, K., Hartmann, J. A., Werts, D., and Zrenner,E. (2001). Histological studies of retinal degeneration and biocompatibility of subretinal implants. Ophthalmologe 98, 364-368.

Kozai, T. D., and Kipke, D. R. (2009). Insertion shuttle with carboxyl terminated self-assembled monolayer coatings for implanting flexible polymer neural probes in the brain. J. Neurosci. Methods 184, 199-205.

Kralik, J. D., Dimitrov, D. F., Krupa, D. J., Katz, D. B., Cohen, D., and Nicolelis, M. A. (2001). Techniques for longterm multisite neuronal ensemble recordings in behaving animals. Methods 25, 121-150.

Lee, H., Bellamkonda, R. V., Sun, W., and Levenston, M. E. (2005). Biomechanical analysis of silicon microelectrode-induced strain in the brain. J. Neural Eng. 2, 81-89.

Leung, B. K., Biran, R., Underwood, C. J., and Tresco, P. A. (2008). Characterization of microglial attachment and cytokine release on biomaterials of differing surface chemistry. Biomaterials 29, 3289-3297.

Liu, X., McCreery, D. B., Carter, R. R., Bullara, L. A., Yuen, T. G., and Agnew, W. F. (1999). Stability of the interface between neural tissue and chronically implanted intracortical microelectrodes. IEEE Trans. Rehabil. Eng. 7,315-326.

Lovat, V., Pantarotto, D., Lagostena, L., Cacciari, B., Grandolfo, M., Righi, M., Spalluto, G., Prato, M., and Ballerini, L. (2005). Carbon nanotube substrates boost neuronal electrical signaling. Nano Lett. 5, 1107-1110.

Ludwig, K.A., Uram, J.D., Yang, J., Martin, D. C., and Kipke, D. R. (2006). Chronic neural recordings using silicon microelectrode arrays electrochemically deposited with a poly(3,4-ethylenedioxythiophene) (PEDOT) film. $J$. Neural Eng. 3, 59-70.

Majji, A. B., Humayun, M. S., Weiland, J. D., Suzuki, S., D'Anna, S. A., and de Juan, E. Jr. (1999). Long-term histological and electrophysiological results of an inactive epiretinal electrode array implantation in dogs. Invest. Ophthalmol. Vis. Sci. 40, 2073-2081.

Mazzatenta,A., Giugliano, M., Campidelli, S., Gambazzi, L., Businaro, L., Markram, H., Prato, M., and Ballerini, L. (2007). Interfacing neurons with carbon nanotubes: electrical signal transfer and synaptic stimulation in cultured brain circuits. J. Neurosci. 27, 6931-6936.

McConnell, G. C., Rees, H. D., Levey, A. I., Gutekunst, C. A., Gross, R. E., and Bellamkonda, R. V. (2009). Implanted neural electrodes cause chronic, local inflammation that is correlated with local neurodegeneration. J. Neural Eng. 6, 56003.
McCreery, D., Agnew, W. F., and Bullara, L. (2002). The effects of prolonged intracortical microstimulation on the excitability of pyramidal tract neurons in the cat. Ann. Biomed. Eng. $30,107-119$.

Merabet, L. B., Rizzo, J. F. III, PascualLeone, A., and Fernandez, E. (2007) 'Who is the ideal candidate?': decisions and issues relating to visual neuroprosthesis development, patient testing and neuroplasticity. J. Neural Eng. 4, S130-S135.

Moxon, K. A., Hallman, S., Aslani, A., Kalkhoran, N. M., and Lelkes, P. I. (2007). Bioactive properties of nanostructured porous silicon for enhancing electrode to neuron interfaces. J. Biomater. Sci. Polym. Ed. 18, 1263-1281.

Musallam, S., Bak, M. J., Troyk, P. R., and Andersen, R. A. (2007). A floating metal microelectrode array for chronic implantation.J. Neurosci. Methods 160 122-127.

Neves, H. (2007). Advances in cerebral probing using modular multifunctional probe arrays. Med. Device Technol. 18, 38-39.

Nicolelis, M., Stambaugh, C. R., Brisben, A., and Laubach, M. (1999). "Methods for simultaneous multisite neural ensemble recordings in behaving primates," in Methods for Neural Ensemble Recordings, ed. M. Nicolelis (Boca Raton, FL: CRC Press LLC), 121-156.

Nicolelis, M. A. (2005). Computing with thalamocortical ensembles during different behavioural states. J. Physiol (Lond.) 566, 37-47.

Nicolelis, M. A., and Lebedev, M. A. (2009). Principles of neural ensemble physiology underlying the operation of brain-machine interfaces. Nat. Rev. Neurosci. 10, 530-540.

Normann, R. A. (2007). Technology insight: future neuroprosthetic therapies for disorders of the nervous system. Nat. Clin. Pract. Neurol. 3, 444-452.

Normann, R. A., Greger, B. A., House, P., Romero, S. F., Pelayo, F., and Fernandez, E. (2009). Toward the development of a cortically based visual neuroprosthesis. J. Neural Eng. 6, 035001

Normann, R.A., Maynard,E.M., Rousche, P. J., and Warren, D. J. (1999). A neural interface for a cortical vision prosthesis. Vision Res. 39, 2577-2587.

Pancrazio, J.J. (2008). Neural interfaces at the nanoscale. Nanomedicine (Lond). 3, 823-830.

Polikov, V. S., Tresco, P. A., and Reichert, W. M. (2005). Response of brain tissue to chronically implanted neural electrodes. J. Neurosci. Methods 148 $1-18$.
Rennaker, R. L., Miller, J., Tang, H., and Wilson, D. A. (2007). Minocycline increases quality and longevity of chronic neural recordings. J. Neural Eng. 4, L1-L5.

Rennaker, R. L., Street, S., Ruyle, A. M., and Sloan, A. M. (2005). A comparison of chronic multi-channel cortical implantation techniques: manual versus mechanical insertion. J. Neurosci. Methods 142, 169-176.

Richardson, R. T., Wise, A. K., Thompson, B. C., Flynn, B. O., Atkinson, P. J., Fretwell, N. J., Fallon, J. B., Wallace, G. G., Shepherd, R. K., Clark, G. M. and O'Leary, S. J. (2009). Polypyrrolecoated electrodes for the delivery of charge and neurotrophins to cochlear neurons. Biomaterials 30, 2614-2624.

Rousche, P.J., Pellinen, D. S., Pivin, D. P. Jr., Williams, J. C., Vetter, R. J., and Kipke, D. R. (2001). Flexible polyimide-based intracortical electrode arrays with bioactive capability. IEEE Trans. Biomed. Eng. 48, 361-371.

Seymour, J. P., and Kipke, D. R. (2007) Neural probe design for reduced tissue encapsulation in CNS. Biomaterials 28, 3594-3607.

Stice, P., Gilletti, A., Panitch, A., and Muthuswamy, J. (2007). Thin microelectrodes reduce GFAP expression in the implant site in rodent somatosensory cortex. J. Neural Eng. 4, 42-53.

Stieglitz, T., and Meyer, J. U. (1999). Implantable microsystems. Polyimidebased neuroprostheses for interfacing nerves. Med. Device Technol. 10, 28-30.

Stieglitz, T., Schuettler, M., and Koch, K. P. (2004). Neural prostheses in clinical applications-trends from precision mechanics towards biomedical microsystems in neurological rehabilitation. Biomed. Tech. (Berl.) 49, 72-77.

Szarowski, D. H., Andersen, M. D., Retterer, S., Spence, A. J., Isaacson, M., Craighead, H. G., Turner, J. N., and Shain, W. (2003). Brain responses to micro-machined silicon devices. Brain Res. 983, 23-35.

Truccolo, W., Friehs, G. M., Donoghue, J. P., and Hochberg, L. R. (2008). Primary motor cortex tuning to intended movement kinematics in humans with tetraplegia. J. Neurosci. 28, 1163-1178.

Turner, J. N., Shain, W., Szarowski, D. H., Andersen, M., Martins, S., Isaacson, M., and Craighead, H. (1999). Cerebral astrocyte response to micromachined silicon implants. Exp. Neurol. 156, 33-49.

Walter, P., Szurman, P., Vobig, M., Berk, H., Ludtke-Handjery, H. C., Richter H., Mittermayer, C., Heimann, K., and Sellhaus, B. (1999). Successful 
long-term implantation of electrically inactive epiretinal microelectrode arrays in rabbits. Retina (Philadelphia, Pa.) 19, 546-552.

Ward,M.P., Rajdev,P.,Ellison, C., and Irazoqui, P. P. (2009). Toward a comparison of microelectrodes for acute and chronic recordings. Brain Res. 1282, 183-200.

Williams, D. F. (2008). On the mechanisms of biocompatibility. Biomaterials 29, 2941-2953.

Wise, K. D. (2005). Silicon microsystems for neuroscience and neural prostheses. IEEE Eng. Med. Biol. Mag. 24, 22-29.

Woodford, B. J., Carter, R. R., McCreery, D., Bullara, L. A., and Agnew, W. F. (1996). Histopathologic and physiologic effects of chronic implantation of microelectrodes in sacral spinal cord of the cat. J. Neuropathol. Exp. Neurol. 55, 982-991.

Zhong, Y., and Bellamkonda, R. V. (2007). Dexamethasone-coated neural probes elicit attenuated inflammatory response and neuronal loss compared to uncoated neural probes. Brain Res. 1148, 15-27.

Conflict of Interest Statement: The authors declare that the research was conducted in the absence of any commercial or financial relationships that could be construed as a potential conflict of interest.

Received: 29 November 2009; paper pending published: 26 February 2010; accepted: 05 May 2010; published online: 28 May 2010.
Citation: Marin Cand Fernández E (2010) Biocompatibility of intracortical microelectrodes: current status and future prospects. Front. Neuroeng. 3:8. doi: 10.3389/ fneng.2010.00008

Copyright (C) 2010 Marin and Fernández. This is an open-access article subject to an exclusive license agreement between the authors and the Frontiers Research Foundation, which permits unrestricted use, distribution, and reproduction in any medium, provided the original authors and source are credited. 